\title{
Relationship between Helicobacter pylori infection and bone mineral density: a retrospective cross-sectional study
}

\author{
Bo-Lin Pan ${ }^{1}$, Chih-Fang Huang ${ }^{1}$, Seng-Kee Chuah², Jui-Chin Chiang ${ }^{1}$ and Song-Seng Loke ${ }^{1 *}$
}

\begin{abstract}
Background: Helicobacter pylori (H. pylori) infection can induce individual inflammatory and immune reactions which associated with extra-digestive disorders. Our aim is to investigate the association between $\mathrm{H}$. pylori infection and bone mineral density.

Methods: This retrospective cross-sectional study was performed by using the data from the health examination database in a medical center of southern Taiwan in 2013. We investigated the relationship between sex, age, body mass index (BMI), waist circumstance, lipid profile, H. pylori infection, the findings of upper gastrointestinal endoscopy and bone mineral density (BMD). Because of nonrandomized assignment and strong confounding effect of age on BMD, the 1:1 propensity score match was applied for age adjustment. The simple and multiple stepwise logistic regression analysis were performed to assess the risk factors of decreased BMD in these well-balanced pairs of participants.

Results: Of the 867 subjects in final analysis with the mean age of $55.9 \pm 11.3$ years, 381 (43.9\%) subjects had H. pylori infection, and 556 (64.1\%) subjects had decreased BMD. In decreased BMD group, the portion of woman was higher than a normal BMD group (37.2\% versus $29.6 \%, P=0.023)$, the age was significantly older $(59.4 \pm 9.8$ versus $49.8 \pm 11.3, p<0.001)$ and BMI was significantly lower $(24.7 \pm 3.5$ versus $25.4 \pm 3.7, p=0.006)$ than the normal BMD group. The prevalence of $H$. pylori infection was $39.9 \%$ and $46.2 \%$ in the normal BMD group and the decreased BMD group respectively $(P=0.071)$. The multivariate analysis which was used for these possible risk factors showed that only advanced age (OR 1.09, 95\% Cl 1.08-1.11, P<0.001), and low BMI (OR 0.91, $95 \% \mathrm{Cl} 0.87-0.95, P<0.001)$ were independently significantly associated with decreased BMD in this nonrandomized study. In the propensity score-matched participants, the multiple stepwise logistic regression analysis revealed $\mathrm{H}$. pylori infection (OR 1.62, 95\% Cl 1.12-2.35, $P=0.011)$ and low BMl (OR 0.92, 95\% Cl 0.87-0.97, $P=0.001)$ were independently significantly associated with decreased BMD.
\end{abstract}

Conclusions: H. pylori infection and low BMI were independently significantly associated with decreased BMD in selected propensity score-matched populations after age adjustment.

Keywords: Helicobacter pylori, Bone mineral density, body mass index, Dual energy X-ray absorptiometry scan, Osteoporosis

\footnotetext{
* Correspondence: loke@adm.cgmh.org.tw

${ }^{1}$ Department of Family Medicine, Kaohsiung Chang Gung Memorial Hospital and Chang Gung University College of Medicine, 123, Dapi Road, Niaosong District, Kaohsiung 833, Taiwan

Full list of author information is available at the end of the article
}

(c) The Author(s). 2018 Open Access This article is distributed under the terms of the Creative Commons Attribution 4.0 International License (http://creativecommons.org/licenses/by/4.0/), which permits unrestricted use, distribution, and reproduction in any medium, provided you give appropriate credit to the original author(s) and the source, provide a link to the Creative Commons license, and indicate if changes were made. The Creative Commons Public Domain Dedication waiver (http://creativecommons.org/publicdomain/zero/1.0/) applies to the data made available in this article, unless otherwise stated. 


\section{Background}

Osteoporosis is a silent health problem characterized by decreased bone mineral density (BMD) with a risk of spine and hip fractures. Approximately half of the hip fractures globally result from osteoporosis. According to data from the National Health Insurance Research Database between 1996 and 2010 in Taiwan, the high annual incidence rate of hip fracture was 472.1 per 100,000 patients per year, higher than that in other Asian countries and even in the world. The in-hospital mortality rates were between 0.85 to $2.26 \%$ [1]. The spine and hip fractures resulted from osteoporosis could induce patients to become bedridden and needing care. Mortality and disability-associated osteoporosis have significant impact on prognosis and are a burden affecting patients, their families, society, and the health system. Early identification of the risk of decreased BMD and osteoporosis is very important. Previous study has revealed that the risk factors of osteoporosis include age, sex, low body mass index (BMI), steroid use and chronic alcohol consumption [2, 3]. Recently, several gastrointestinal diseases such as inflammatory bowel disease, peptic ulcer disease and atrophic gastritis have been suspected of being risk factors for osteoporosis [4-6].

Helicobacter pylori (H. pylori) infection is strongly associated with chronic gastritis, peptic ulcers, gastric cancer, and mucosa-associated lymphoid tissue lymphoma [7]. Several studies have reported $H$. pylori also plays a role in extra-digestive disorders including cardiovascular, neurological, skin disease and diabetes mellitus [8].

H. pylori infection can induce individual inflammatory and immune reactions, which can regulate bone turnover. Several research studies have reported that $H$. pylori infection is a risk factor of osteoporosis $[9,10]$, but this finding is controversial in other studies $[11,12]$. To our knowledge, only one study has evaluated the relationship between $H$. pylori infection and osteoporosis in Taiwan, but the result was only found in elderly females [10]. Therefore, we aimed to investigate whether $H$. pylori infection was associated with decreased BMD in a general population undergoing routine health examination in Taiwan.

\section{Methods}

\section{Subjects}

This retrospective cross-sectional study was performed by using the data from the health examination database in a medical center of the southern Taiwan. We included subjects who were aged greater than 20 years, had undergone upper gastrointestinal endoscopy with the Campylobacter-like organism test (CLO test) and dual energy X-ray absorptiometry scan (DEXA) from January 2013 to December 2013. Subjects with missing data and gastric cancer were excluded. The study was approved by the Chang Gung Medical Foundation Institutional Review Board (IRB No.: 201701187B0).
Since this is a retrospective study, a written consent is waived by an IRB and is deemed unnecessary.

The medical records showed information on sex, age, waist circumstance, lipid profile, $H$. pylori testing, BMD, and the findings of upper gastrointestinal endoscopy. BMI was calculated as the weight in kilograms divided by the height in meters squared. Peptic ulcer or gastro-esophageal reflux disease (GERD) were diagnosed by upper gastrointestinal endoscopy.

\section{Definition of $\boldsymbol{H}$. pylori infection}

The present of $H$. pylori infection was determined by CLO test via upper gastrointestinal endoscopy. CLO test is a rapid diagnostic test and is performed during upper gastrointestinal endoscopy. A biopsy of mucosa from the stomach is placed into the medium consisting of urea and an indicator. If $H$. pylori is present, the urease produced by $H$. pylori converts urea to ammonia, which increases the $\mathrm{pH}$ of the medium and then changes its color from yellow to red. In this health examination, all examinations were self-paid, CLO test was performed not only for peptic ulcer, but also performed under subjects request although no peptic ulcer was found by upper gastrointestinal endoscopy.

\section{Determination of bone mineral density}

BMD was measured by DEXA. The $T$-score is the number of standard deviations by which a given measurement differs from the mean for a normal young adult reference population. According to the World Health Organization definition [13], osteoporosis is defined as $T$-score $\leq-2.5$, and the osteopenia is defined as a T-score between -1 and -2.5 . Decreased BMD in the present study included osteoporosis and osteopenia.

\section{Statistical analysis}

All data are described as the mean \pm standard deviation for continuous variables and as numbers and percentages for categorical variables. SPSS software version 19.0 (IBM Corp., Armonk, NY, USA)) was used for the statistical analysis. The characteristics of subjects were compared by $x^{2}$-test for the categorical variables and Student's t-test for the continuous variables. Univariate logistic regression analysis and multivariate logistic regression analysis were conducted to analyze the odds ratio (OR) of significant factors associated with decreased BMD. Besides, to minimize confounding effect of age due to nonrandomized assignment, propensity scores were calculated using a logistic regression model and the covariate, age. A 1:1 matched study group was created by the Greedy method with NCSS software (NCSS 10, NCSS Statistical software, Kaysville, Utah). After adjusting the age, univariate logistic regression analysis and multivariate logistic regression analysis were used to evaluate factors associated with 
decreased BMD. The strength of association was reported as OR with $95 \%$ confidential interval (CI) and $P$ values. All statistical assessments were two-sided and considered significant if $P<0.05$.

\section{Results}

Prevalence of $H$. pylori infection and decreased BMD

We enroll 942 subjects who participated in the health examination and underwent biochemistry blood examination, upper gastrointestinal endoscopy with CLO test and the bone mineral density examinations. Seventy-five subjects were excluded due to missing data in some biochemical variables. Of the 867 subjects in final analysis with the mean age of $55.9 \pm 11.3$ years, $381(43.9 \%)$ subjects had H. pylori infection, and 556 (64.1\%) subjects had decreased BMD. The numbers of female and male subjects were $299(34.5 \%)$ and 568 (65.5\%). Table 1 shows the baseline characteristics of all participants.

\section{Differences between the subjects with normal and decreased BMD}

Table 2 compares the characteristics between the normal $\mathrm{BMD}$ group and the decreased BMD group. In decreased BMD group, the portion of woman was higher than a normal BMD group $(37.2 \%$ versus $29.6 \%, P=0$. $023)$, the age was significantly older $(59.4 \pm 9.8$ versus $49.8 \pm 11.3, p<0.001)$ and BMI was significantly lower $(24.7 \pm 3.5$ versus $25.4 \pm 3.7, p=0.006)$ than the normal BMD group. Besides, lipid profiles except for triglyceride were significantly higher than in the normal BMD group. Comparing the gastrointestinal disorder between these

Table 1 Baseline characteristics

\begin{tabular}{ll}
\hline & $\begin{array}{l}\text { Participants } \\
N=867\end{array}$ \\
\hline Sex & \\
$\quad$ Female $\mathrm{n}, \%$ & $299(34.5 \%)$ \\
$\quad$ Male $\mathrm{n}, \%$ & $568(65.5 \%)$ \\
Age (years old) & $55.9 \pm 11.3$ \\
BMl(Kg/m $\left.{ }^{2}\right)$ & $24.9 \pm 3.6$ \\
Waist circumference(cm) & $85.1 \pm 10.4$ \\
Total cholesterol(mg/dl) & $195.7 \pm 35.7$ \\
HDL cholesterol(mg/dl) & $56.5 \pm 15.6$ \\
Triglyceride(mg/dl) & $131.9 \pm 84.7$ \\
LDL cholesterol(mg/dl) & $113.4 \pm 32.4$ \\
Decreased BMD, $\mathrm{n} \%$ & $556(64.1 \%)$ \\
Peptic ulcer $\mathrm{n}, \%$ & $351(40.5 \%)$ \\
GERD n, \% & $187(21.6 \%)$ \\
H. pylori infection n, \% & $381(43.9 \%)$ \\
\hline BMI
\end{tabular}

$B M I$ body mass index, $L D L$ low-density lipoprotein, $H D L$ high-density lipoprotein, $B M D$ bone mineral density, GERD gastro-esophageal reflux disease, $\mathrm{H}$. pylori Helicobacter pylori
Table 2 Differences between normal BMD group and decreased BMD group

\begin{tabular}{|c|c|c|c|}
\hline & $\begin{array}{l}\text { Normal BMD } \\
n=311\end{array}$ & $\begin{array}{l}\text { Decreased BMD } \\
n=556\end{array}$ & $p$ value \\
\hline Sex & & & $0.023^{*}$ \\
\hline Male n, \% & $219(70.4 \%)$ & $349(62.8 \%)$ & \\
\hline Female $n, \%$ & $92(29.6 \%)$ & $207(37.2 \%)$ & \\
\hline Age (years old) & $49.8 \pm 11.3$ & $59.4 \pm 9.8$ & $<0.001^{*}$ \\
\hline $\mathrm{BMI}\left(\mathrm{Kg} / \mathrm{m}^{2}\right)$ & $25.4 \pm 3.7$ & $24.7 \pm 3.5$ & $0.006^{*}$ \\
\hline Waist circumference $(\mathrm{cm})$ & $85.9 \pm 10.1$ & $84.7 \pm 10.5$ & 0.08 \\
\hline Total cholesterol(mg/dl) & $191.2 \pm 34.5$ & $198.2 \pm 36.2$ & $0.005^{*}$ \\
\hline HDL cholesterol(mg/dl) & $54.4 \pm 14.9$ & $57.8 \pm 15.8$ & $0.002^{*}$ \\
\hline Triglyceride(mg/dl) & $136.8 \pm 83.8$ & $129.2 \pm 85.2$ & 0.205 \\
\hline LDL cholesterol(mg/dl) & $110.0 \pm 31.5$ & $115.3 \pm 32.7$ & $0.021^{*}$ \\
\hline Peptic ulcer n, \% & $111(35.7 \%)$ & $240(43.2 \%)$ & $0.032^{*}$ \\
\hline GERD n, \% & $69(22.2 \%)$ & $118(21.2 \%)$ & 0.741 \\
\hline H. pylori infection $n, \%$ & 124(39.9\%) & $257(46.2 \%)$ & 0.071 \\
\hline
\end{tabular}

two groups, the prevalence of peptic ulcer was significantly higher in the decreased BMD group $(p=0.032)$, but the GERD was lower in the decreased BMD group $(p=0.741)$. The prevalence of $H$. pylori infection was $39.9 \%$ and $46.2 \%$ in the normal BMD group and decreased BMD group respectively $(P=0.071)$.

Simple and multiple stepwise logistic regression analyses of variables associated with decreased BMD

Simple logistic regression (Table 3) showed decreased BMD was significantly associated with the female gender (OR $0.71,95 \%$ CI $0.53-0.94, P=0.023$ ), advanced age (OR 1.09, 95\% CI 1.07-1.10, $P<0.001$ ), low BMI (OR 0.95, 95\% CI 0.91-0.99, $P=0.006$ ), total cholesterol (OR 1.01, 95\% CI 1.00-1.01, $P=0.005)$, low-density lipoprotein (LDL) cholesterol (OR 1.01, 95\% CI 1.00-1.01, $P=0.021$ ), high-density lipoprotein (HDL) cholesterol (OR 1.02, 95\% CI 1.00-1.02, $P=0.002$ ), and peptic ulcer disease (OR 1.37, $95 \%$ CI 1.03-1.82, $P=0.032$ ). The multivariate analysis that was conducted with these risk factors revealed that only advanced age (OR 1.09, 95\% CI 1.08-1.11, $P<0.001$ ), and low BMI (OR 0.91, 95\% CI 0.87-0.95, $P<0.001$ ) were independently significantly associated with decreased BMD. The H. pylori infection was not independently significantly associated with decreased BMD (OR 1.30, 95\% CI $0.98-1.71, P=0.071$ ).

Because the confounding effect of age was strong with nonrandomized assignment, propensity score-matching was used for age adjustment. The 234 well-balanced pairs of participants, with a 1:1 ratio after propensity score matching of age, were evaluated for risk factor of decreased 
Table 3 Regression analysis for association of decreased BMD with different variables

\begin{tabular}{llll}
\hline Variables & $\begin{array}{l}\text { Simple logistic regression } \\
\text { OR }(95 \% \mathrm{Cl})\end{array}$ & $p$ value & $\begin{array}{l}\text { multiple stepwise logistic regression } \\
\text { OR }(95 \% \mathrm{Cl})\end{array}$ \\
\hline Sex & $0.71(0.53-0.94)$ & $0.023^{*}$ & \\
Age & $1.09(1.07-1.10)$ & $<0.001^{*}$ & $1.09(1.08-1.11)$ \\
BMI & $0.95(0.91-0.99)$ & $0.006^{*}$ & $0.91(0.87-0.95)$ \\
Waist circumference & $0.99(0.98-1.00)$ & 0.08 & \\
Total cholesterol & $1.01(1.00-1.01)$ & $0.005^{*}$ & \\
HDL cholesterol & $1.02(1.00-1.02)$ & $0.002^{*}$ & \\
TG & $1.00(0.99-1.001)$ & 0.207 & \\
LDL cholesterol & $1.01(1.00-1.01)$ & $0.021^{*}$ & \\
Peptic ulcer & $1.37(1.03-1.82)$ & $0.032^{*}$ & \\
GERD & $0.95(0.68-1.32)$ & 0.74 & \\
H. pylori infection & $1.30(0.98-1.71)$ & 0.071 & \\
\hline
\end{tabular}

*Indicates a significant difference, $p<0.05$

$B M I$ body mass index, $L D L$, low-density lipoprotein, $H D L$ high-density lipoprotein, $B M D$ bone mineral density, GERD gastro-esophageal reflux disease, $H$. pylori Helicobacter pylori

BMD. In these propensity score-matched participants, the mean age was $53.3 \pm 10.35$ years in the normal BMD group and $53.4 \pm 10.33$ years in the decreased BMD group. There was no significant difference in age between the two groups $(p=0.961)$. The covariates of these well-balanced pairs of participants were conducted for simple and multiple stepwise logistic regression analysis. In simple logistic regression, the BMI (OR 0.92, 95\% CI 0.87-0.97, $P=0.001$ ), waist circumstance (OR 0.98, 95\% CI 0.96-0.99, $P=0.006$ ) and H. pylori infection (OR 1.60, 95\% CI 1.11-2.30, $P=0.012$ ) were significantly associated with decreased BMD. In multivariate analysis, $H$. pylori infection (OR 1.62, 95\% CI $1.12-2.35, P=0.011$ ) and BMI (OR 0.92, 95\% CI 0.87-0.97, $P=0.001)$ were independent significant risk factors of decreased BMD without confounding effect of age (Table 4).

\section{Discussion}

The main finding of this retrospective cross-sectional study revealed that advanced age and low BMI were significant risk factors of decreased BMD in the nonrandomized assignment. In addition, $H$. pylori infection was significantly associated with decreased BMD in selected propensity score-matched participants with respect to age. In other words, $H$. pylori infection was a risk factor of decreased $\mathrm{BMD}$ without the confounding effect of age, because the age corresponded to an increase in risk of osteoporosis [2].

This result was compatible with the past studies regarding the association between $H$. pylori infection and decreased BMD [10, 12, 14]. Several possible mechanisms might explain this finding. First, $H$. pylori infection may result in chronic gastritis and induce systemic

Table 4 Regression analysis for association between decreased BMD and different variables after propensity score matching

\begin{tabular}{llll}
\hline Variables & $\begin{array}{l}\text { Simple logistic regression } \\
\text { OR }(95 \% \mathrm{Cl})\end{array}$ & $p$ value & $\begin{array}{c}\text { Multiple stepwise logistic regression } \\
\text { OR }(95 \% \mathrm{Cl})\end{array}$ \\
\hline Sex & $0.80(0.54-1.19)$ & 0.27 & $0.92(0.87-0.97)$ \\
BMI & $0.92(0.87-0.97)$ & $0.001^{*}$ & $0.001^{*}$ \\
Waist circumference & $0.98(0.96-0.99)$ & $0.006^{*}$ & \\
Total cholesterol & $1.00(0.99-1.01)$ & 0.257 & \\
HDL & $1.01(1.00-1.02)$ & 0.082 & \\
TG & $1.00(0.99-1.001)$ & 0.672 & \\
LDL & $1.00(0.99-1.01)$ & 0.425 & \\
Peptic ulcer & $1.10(0.75-1.60)$ & 0.632 & $1.62(1.12-2.35)$ \\
GERD & $0.88(0.56-1.38)$ & 0.564 & $0.011^{*}$ \\
H. pylori infection & $1.60(1.11-2.30)$ & $0.012^{*}$ & \\
\hline
\end{tabular}

*Indicates a significant difference, $p<0.05$

$B M I$ body mass index, $L D L$ low-density lipoprotein, $H D L$ high-density lipoprotein, $B M D$ bone mineral density, GERD gastro-esophageal reflux disease, $H$. pylori Helicobacter pylori 
inflammation with the release of cytokines, including tumor necrosis factor- , interleukin-1 and interleukin-6 [15]. These inflammatory cytokines are known to result in bone resorption, so $H$. pylori infection may affect bone turnover indirectly [16]. The second mechanism was that chronic $H$. pylori infection might cause the gastric mucosal atrophy which would decrease acid secretion. The hypochlorhydric stomach affects calcium absorption, calcium homeostasis and bone mass [17]. In addition, the low serum vitamin B12 level was found in the patients with $H$. pylori infection [18]. If the serum vitamin B12 levels are low, the folate becomes trapped as methyltetrahydrofolate and then interrupts for folate-related DNA synthesis. This reaction is an important factor of bone remodeling, so the low level of vitamin B12 may result in decreased BMD [19].

Besides, $H$. pylori infection was treated with the eradication therapy as triple or quadruple regimen, such as a proton pump inhibitor, clarithromycin, amoxicillin or tetracycline, and metronidazole, with or without bismuth. One study described cytokine gene expression as significantly decreasing after $H$. pylori was eradicated [20]. In a meta-analysis, mucosal atrophy of the stomach was improved after successful eradication therapy [21]. Because the cytokines result in bone resorption and gastric mucosal atrophy affects calcium metabolism, the improvement of gastric mucosa and decreased inflammatory cytokine by successful eradication therapy might be able to decrease the incidence of osteoporosis. Two studies support this hypothesis. Hong-Mo Shih et al. reported the incidence of osteoporosis relatively reduced in early eradication of $H$. pylori group, compared to the late eradication group by an analysis from the National Health Insurance Database in Taiwan [22]. In Japan, the success of H. pylori eradication may contribute to decrease the risk of osteoporosis. In our study, we found that $H$. pylori infection was independently significantly associated with decreased BMD after age adjustment, but eradication of H. pylori was not recorded in this health examination database. A further prospective cohort study is necessary to confirm that BMD would be improved after triple or quadruple $H$. pylori eradication therapy.

Advanced age and low BMI are well-known risk factors of osteoporosis and bone fracture [2]. From the National Health and Nutrition Examination Survey (NHANES) 2005-2006 in US, 49\% of US women age 50 years and older had decreased BMD, and $30 \%$ of older men had decreased BMD [23]. The prevalence rates of BMD from Nutrition and Health Survey in Taiwan 2005-2008 was higher in advanced age. In females, the prevalence rate was $50.3 \%$ in the 60 years and older group, and increased to $63.7 \%$ in the 70 years and older group. In male, the prevalence rate was $18.6 \%$ in the 60 years and older group, and increased to $45.4 \%$ in those aged 70 years and older
[24]. One research showed that the weight loss appears to increase the rate of hip bone loss, even in obese men undergoing voluntary weight reduction [25]. This present study reported a similar result where the advanced age and low BMI had the strong association with the decreased BMD.

Several researches have reported that the GERD was associated with osteoporosis. However, the vertebral fractures or kyphosis were also found in these studies [26-28]. In the present study, the GERD was not significantly associated with decreased BMD. Because this study was derived from the health examinations, these subjects may be healthy relatively without any bone deformities. Besides, two studies revealed peptic ulcer disease was an independent risk factor for osteoporosis [6, 29]. The mechanism of this association was not clear. The malabsorption of calcium and macroelements due to the defective stomach and duodenal epithelium in which inflammation at these sites results may play a role in bone metabolism. Our result showed the peptic ulcer was not a risk factor of decreased BMD, but it tended to be associated with decreased BMD in univariate logistic regression analysis. The disorder of the small intestine may affect the absorption of these substances more than disorders of the stomach and duodenum.

There were several limitations in our study. First, our study was conducted in a single hospital, which might not be representative of other settings. Second, retrospective studies based on abstraction of medical records are constrained by the accuracy and completeness of such records. Third, some laboratory data, including serum calcium, serum phosphorus, serum specific alkaline phosphatase or serum vitamin D level were unavailable, because this study was retrospective from the health examination.

\section{Conclusions}

In conclusion, advanced age and low BMI were independent significant risk factors of decreased BMD in this retrospective study. Besides low BMI, $H$. pylori infection was independently significantly associated with decreased BMD in selected propensity score-matched participants with respect to age. Further prospective cohort studies including the potential important factors are required to confirm this association in the Taiwan population.

\section{Abbreviations}

BMD: Bone mineral density; BMI: Body mass index; Cl: Confidential interval; CLO test: Campylobacter-like organism test; DEXA: Dual energy X-ray absorptiometry scan; GERD: Gastro-esophageal reflux disease; H. pylori: Helicobacter pylori; HDL: High-density lipoprotein; LDL: Low-density lipoprotein

\section{Acknowledgments}

We appreciated the Biostatistics Center, Kaohsiung Chang Gung Memorial Hospital for statistics work.

Funding

This study was not supported by any grant. 


\section{Availability of data and materials}

The datasets used and analysed during the current study will be available from the corresponding author on reasonable request.

\section{Authors' contributions}

PBL analyzed the data and drafted the paper; HCF, CSK, CJC performed the research; LSS designed the research and revised the paper. All authors read and approved the final manuscript.

\section{Ethics approval and consent to participate}

This study was approved by the Chang Gung Medical Foundation Institutional Review Board (IRB No.: 201701187B0). Since this is a retrospective study, a written consent is waived by an IRB and is deemed unnecessary.

\section{Competing interests}

CSK is a member of the editorial board of this journal. All other authors declare that they have no competing interests.

\section{Publisher's Note}

Springer Nature remains neutral with regard to jurisdictional claims in published maps and institutional affiliations.

\section{Author details}

'Department of Family Medicine, Kaohsiung Chang Gung Memorial Hospital and Chang Gung University College of Medicine, 123, Dapi Road, Niaosong District, Kaohsiung 833, Taiwan. ${ }^{2}$ Division of Hepatogastroenterology, Department of Internal Medicine, Kaohsiung Chang Gung Memorial Hospital and Chang Gung University College of Medicine, 123, Dapi Road, Niaosong District, Kaohsiung 833, Taiwan.

Received: 10 December 2017 Accepted: 17 April 2018

Published online: 24 April 2018

\section{References}

1. Wu TY, Hu HY, Lin SY, Chie WC, Yang RS, Liaw CK. Trends in hip fracture rates in Taiwan: a nationwide study from 1996 to 2010. Osteoporos Int. 2017;28(2):653-65.

2. Nih Consensus Development Panel on Osteoporosis Prevention D, Therapy. Osteoporosis prevention, diagnosis, and therapy. JAMA. 2001;285(6):785-95.

3. Kouda K, Iki M, Fujita Y, Tamaki J, Yura A, Kadowaki E, Sato Y, Moon JS, Morikawa M, Tomioka K, et al. Alcohol intake and bone status in elderly Japanese men: baseline data from the Fujiwara-kyo osteoporosis risk in men (FORMEN) study. Bone. 2011:49(2):275-80.

4. Ali T, Lam D, Bronze MS, Humphrey MB. Osteoporosis in inflammatory bowel disease. Am J Med. 2009;122(7):599-604.

5. Kim HW, Kim YH, Han K, Nam GE, Kim GS, Han BD, Lee A, Ahn JY, Ko BJ. Atrophic gastritis: a related factor for osteoporosis in elderly women. PLoS One. 2014;9(7):e101852.

6. Sawicki A, Regula A, Godwod K, Debinski A. Peptic ulcer disease and calcium intake as risk factors of osteoporosis in women. Osteoporos Int. 2003;14(12):983-6.

7. Atherton JC. The pathogenesis of Helicobacter pylori-induced gastro-duodenal diseases. Annu Rev Pathol. 2006;1:63-96.

8. Goni E, Franceschi F. Helicobacter pylori and extragastric diseases. Helicobacter. 2016;21(Suppl 1):45-8.

9. Asaoka D, Nagahara A, Shimada Y, Matsumoto K, Ueyama H, Matsumoto K, Nakagawa Y, Takeda T, Tanaka I, Sasaki H, et al. Risk factors for osteoporosis in Japan: is it associated with Helicobacter pylori? Ther Clin Risk Manag. 2015;11:381-91

10. Lin SC, Koo M, Tsai KW. Association between Helicobacter pylori infection and risk of osteoporosis in elderly Taiwanese women with upper gastrointestinal diseases: a retrospective patient record review. Gastroenterol Res Pract. 2014;2014:814756.

11. Kakehasi AM, Mendes CM, Coelho LG, Castro LP, Barbosa AJ. The presence of Helicobacter pylori in postmenopausal women is not a factor to the decrease of bone mineral density. Arq Gastroenterol. 2007:44(3):266-70.

12. Fotouk-Kiai M, Hoseini SR, Meftah N, Ghadimi R, Bijani A, Noreddini H, Nematollahi H, Shokri-Shirvani J. Relationship between Helicobacter pylori infection (HP) and bone mineral density (BMD) in elderly people. Caspian J Intern Med. 2015;6(2):62-6.
13. Kanis JA. Assessment of fracture risk and its application to screening for postmenopausal osteoporosis: synopsis of a WHO report. WHO Study Group Osteoporos Int. 1994;4(6):368-81.

14. Asaoka D, Nagahara A, Hojo M, Sasaki H, Shimada Y, Yoshizawa T, Osada T, Watanabe $\mathrm{S}$. The relationship between $\mathrm{H}$. Pylori infection and osteoporosis in Japan. Gastroenterol Res Pract. 2014;2014:340765.

15. Noach LA, Bosma NB, Jansen J, Hoek FJ, van Deventer SJ, Tytgat GN. Mucosal tumor necrosis factor-alpha, interleukin-1 beta, and interleukin-8 production in patients with Helicobacter pylori infection. Scand J Gastroenterol. 1994;29(5):425-9.

16. Pacifici R. Cytokines, estrogen, and postmenopausal osteoporosis-the second decade. Endocrinology. 1998;139(6):2659-61.

17. Kakehasi AM, Rodrigues CB, Carvalho AV, Barbosa AJ. Chronic gastritis and bone mineral density in women. Dig Dis Sci. 2009;54(4):819-24.

18. Kalkan C, Karakaya F, Tuzun A, Gencturk ZB, Soykan I. Factors related to low serum vitamin B12 levels in elderly patients with non-atrophic gastritis in contrast to patients with normal vitamin B12 levels. Geriatr Gerontol Int. 2016;16(6):686-92

19. Tucker KL, Hannan MT, Qiao N, Jacques PF, Selhub J, Cupples LA, Kiel DP. Low plasma vitamin $\mathrm{B} 12$ is associated with lower BMD: the Framingham osteoporosis study. J Bone Miner Res. 2005;20(1):152-8.

20. Moss S, Legon S, Davies J, Calam J. Cytokine gene expression in Helicobacter pylori associated antral gastritis. Gut. 1994;35(11):1567-70.

21. Rokkas T, Pistiolas D, Sechopoulos P, Robotis I, Margantinis G. The long-term impact of Helicobacter pylori eradication on gastric histology: a systematic review and meta-analysis. Helicobacter. 2007;12(Suppl 2):32-8.

22. Shih H-M, Hsu T-Y, Chen C-Y, Lin C-L, Kao C-H, Chen C-H, Yang T-Y, Chen W-K. Analysis of patients with Helicobacter pylori infection and the subsequent risk of developing osteoporosis after eradication therapy: a Nationwide population-based cohort study. PLoS One. 2016;11(9):e0162645.

23. Looker AC, Melton LJ 3rd, Harris TB, Borrud LG, Shepherd JA. Prevalence and trends in low femur bone density among older US adults: NHANES 2005-2006 compared with NHANES III. J Bone Miner Res. 2010:25(1):64-71.

24. Lin YC, Pan WH. Bone mineral density in adults in Taiwan: results of the nutrition and health survey in Taiwan 2005-2008 (NAHSIT 2005-2008). Asia Pac J Clin Nutr. 2011;20(2):283-91.

25. Ensrud KE, Fullman RL, Barrett-Connor E, Cauley JA, Stefanick ML, Fink HA, Lewis $C E$, Orwoll E. Osteoporotic fractures in men study research G: voluntary weight reduction in older men increases hip bone loss: the osteoporotic fractures in men study. J Clin Endocrinol Metab. 2005;90(4):1998-2004.

26. Miyakoshi N, Kasukawa Y, Sasaki H, Kamo K, Shimada Y. Impact of spinal kyphosis on gastroesophageal reflux disease symptoms in patients with osteoporosis. Osteoporos Int. 2009;20(7):1193-8.

27. Sugimoto M, Hasegawa T, Nishino M, Sahara S, Uotani T, Ichikawa H, Kagami T, Sugimoto K, Yamato Y, Togawa D, et al. Improvement of gastroesophageal reflux disease in Japanese patients with spinal kyphotic deformity who underwent surgical spinal correction. Dig Endosc. 2016;28(1):50-8.

28. Yamane $Y$, Yamaguchi $T$, Tsumori M, Yamauchi M, Yano S, Yamamoto M, Honda C, Kinoshita Y, Sugimoto T. Elcatonin is effective for lower back pain and the symptoms of gastroesophageal reflux disease in elderly osteoporotic patients with kyphosis. Geriatr Gerontol Int. 2011;11(2):215-20.

29. Wu CH, Tung YC, Chai CY, Lu YY, Su YF, Tsai TH, Kuo KL, Lin CL. Increased risk of osteoporosis in patients with peptic ulcer disease: a Nationwide population-based study. Medicine (Baltimore). 2016;95(16):e3309.

\section{Ready to submit your research? Choose BMC and benefit from}

- fast, convenient online submission

- thorough peer review by experienced researchers in your field

- rapid publication on acceptance

- support for research data, including large and complex data types

- gold Open Access which fosters wider collaboration and increased citations

- maximum visibility for your research: over $100 \mathrm{M}$ website views per year

\section{At BMC, research is always in progress.}

Learn more biomedcentral.com/submissions 